\title{
Acidose tubular renal distal associada à paralisia periódica hipocalêmica
}

\section{Distal renal tubular acidosis associated with hypokalemic periodic paralysis}

\author{
Elana Couto de Alencar Daniel ${ }^{1}$. Elizabeth de Francesco Daher². \\ 1 Médica, Universidade Federal do Ceará (UFC), Residência em Clínica Médica, Hospital Universitário Walter Cantídio; Psicóloga, \\ Especialista em Tanatologia , Faculdade de Tecnologia do Nordeste, Fortaleza, Ceará, Brasil. 2 Doutora em Medicina Nefrologia \\ pela Universidade de São Paulo (USP), Professora Associada IV, Universidade Federal do Ceará (UFC), Coordenadora do Curso \\ de Medicina e do Internato da Faculdade de Medicina da UFC, Preceptora do Hospital Geral de Fortaleza e Voluntary Assistance \\ Professor, University of Miami. Professora do Curso da Pós-Graduação em Ciências Médicas do Departamento de Medicina \\ Clínica da Faculdade de Medicina da UFC, Bolsista Pesquisadora do CNPQ Nível 2, Fortaleza, Ceará, Brasil.
}

\section{RESUMO}

A acidose tubular renal distal (tipo I) consiste em uma doença de curso crônico na qual ocorre secreção inadequada de prótons no túbulo distal. A depleção de potássio resultante dessa patologia, se não tratada precocemente, pode acarretar paralisia hipocalêmica em raros casos, podendo inclusive levar ao óbito do paciente por complicações decorrentes dessa condição.

O presente artigo visa relatar o caso de uma paciente acompanhada no Serviço de Nefrologia do Hospital Universitário Walter Cantídio. Essa jovem possui acidose tubular renal distal, tendo evoluído com paralisia hipocalêmica. Ao longo de dez anos de acompanhamento no nosso serviço, a paciente apresentou vários episódios de paralisia hipocalêmica, alguns dos quais associados a extrassístoles ventriculares, devido à dificuldade de aderência ao tratamento. Foi evidenciada, ainda, nefrocalcinose, nefrolitíase, acidose metabólica hiperclorêmica e pH urinário inadequadamente alto.

Palavras-chave: Acidose tubular renal distal. Hipopotassemia. Paralisia periódica hipopotassêmica.

\section{ABSTRACT}

The distal renal tubular acidosis (type I) consists of a chronic course of disease in which inappropriate secretion of protons occurs in the distal tubule. The resulting depletion of potassium in that condition, if not treated early, can cause hypokalemic paralysis in rare cases, and it can even lead to the patient's death from complications of this condition.

This article aims to report the case of a patient accompanied at the Nephrology Service of the Hospital Universitário Walter Cantídio. This young woman has distal renal tubular acidosis, having evolved with hypokalemic paralysis. Over ten years of follow-up in our hospital, the patient had several episodes of hypokalemic paralysis, some of which associated with ventricular extrasystoles because of the difficulty of compliance to the treatment. It was evident, yet, nephrocalcinosis, nephrolithiasis, hyperchloremic metabolic acidosis and inappropriately high urinary $\mathrm{pH}$.

Keywords: Distal renal tubular acidosis. Hypokalemia. Hypokalemic periodic paralysis.

Autor correspondente: Elana Couto de Alencar Daniel, Rua Dr. José Lourenço, 2510, apto 1202, Fortaleza, Ceará. Telefone: +55 85 $3246-0219$. E-mail: elana_alencar@hotmail.com

Conflito de interesses: Não há qualquer conflito de interesses por parte de qualquer um dos autores.

Recebido em: 10 Jun 2015; Revisado em: 14 Set 2015; Aceito em: 19 Out 2015. 


\section{INTRODUÇÃO}

A acidose tubular renal corresponde a um espectro de doenças, sendo identificada por acidose metabólica secundária à incapacidade de reabsorção tubular de bicarbonato e/ou de excreção urinária de hidrogênio. $\mathrm{Na}$ acidose tubular renal tipo I (distal), percebe-se secreção inadequada de hidrogênio no túbulo distal. ${ }^{1} \mathrm{Na}$ acidose tubular renal tipo II (proximal), ocorre deficiência na reabsorção do bicarbonato no túbulo proximal. ${ }^{1} \mathrm{Na}$ acidose tubular renal tipo IV, há uma redução da resposta tubular à ação dos mineralocorticoides. ${ }^{1}$

A acidose tubular renal distal (ATRd) caracteriza-se, sobretudo, por uma deficiência na acidificação urinária na região distal do néfron na presença de uma taxa de filtração glomerular normal. ${ }^{2}$ Essa patologia costuma cursar com acidose metabólica hiperclorêmica, $\mathrm{pH}$ urinário inadequadamente alto na presença de acidose sistêmica, hipocalemia, nefrocalcinose e nefrolitíase. ${ }^{3}$

De fato, a hipocalemia é encontrada em cerca de 28 a $53 \%$ dos pacientes com ATRd. ${ }^{4}$ Embora a ATRd seja em geral assintomática, raros indivíduos apresentam paralisia hipocalêmica no curso de sua doença. ${ }^{4}$ Esta é caracterizada por uma paralisia flácida aguda associada à hipocalemia, podendo acarretar complicações que ameaçam a vida, tais como arritmia cardíaca e comprometimento respiratório. ${ }^{5} \mathrm{~A}$ paralisia hipocalêmica periódica corresponde a ataques recorrentes de fraqueza na presença de potássio sérico abaixo de $3,5 \mathrm{mEq} / \mathrm{L} .{ }^{5}$

\section{CASO CLÍNICO}

Paciente de 18 anos abriu o quadro com paresia e parestesia em membros inferiores, o que a impossibilitou de sair do leito. Não buscou atendimento médico, tendo obtido resolução espontânea dos sintomas no dia seguinte. Aproximadamente oito meses após, apresentou novo episódio de parestesia em membros inferiores. Nesta ocasião, descobriu que estava grávida. Novamente, os sintomas regrediram espontaneamente.

No oitavo mês de gestação, foi admitida na emergência obstétrica com lombalgia intensa bilateral, sem irradiação, associada à parestesia e paresia em membros inferiores e superiores, hipocalemia, extrassístoles ventriculares e acidose metabólica com ânion gap normal. A gestação foi resolvida por via vaginal, tendo sido levantada a hipótese diagnóstica de acidose tubular renal.

Foi, então, encaminhada ao serviço de Nefrologia do HUWC para dar continuidade ao acompanhamento. As principais causas de acidose metabólica hiperclorêmica são acidose tubular renal e a diarreia. A história da paciente condiz com o primeiro diagnóstico, pois ela apresentava $\mathrm{pH}$ urinário elevado na presença de acidose sistêmica, demonstrando uma incapacidade de acidificar a urina. Assim, foi estabelecido o diagnóstico de acidose tubular renal tipo I.

$\mathrm{Na}$ consulta inicial, a paciente persistia referindo paresia em membros inferiores, e o potássio sérico estava em $2,7 \mathrm{mmol} / \mathrm{L}$. Foi prescrita reposição oral de potássio ( $\mathrm{KCl}$ xarope $6 \% 20 \mathrm{~mL}$ de $12 / 12 \mathrm{~h}$ e citrato de potássio $20 \mathrm{mEq}$ de $8 / 8 \mathrm{~h}$ ), bicarbonato de sódio (1 colher de sopa, 3 vezes ao dia) e carbonato de cálcio/vitamina D (500 mg/400 UI, 2 vezes ao dia).

A paciente evoluiu apresentando crises de parestesia e paresia em membros inferiores, por vezes impedindo a deambulação. Foi detectada dificuldade econômica em adquirir a medicação prescrita. A Paciente apresentava, ainda, extrassístoles assintomáticas, sendo indicada internação hospitalar para estabilização do quadro (potássio sérico: 2,9 $\mathrm{mmol} / \mathrm{L}$ ), onde fez reposição endovenosa de potássio com $\mathrm{KCl} 10 \%$. Paulatinamente, os níveis séricos de potássio se normalizaram e ritmo cardíaco voltou a ser regular. Antes da alta hospitalar, foi acrescentado à prescrição espironolactona $100 \mathrm{mg} / \mathrm{dia}$. Durante esse internamento, após três anos do diagnóstico inicial, foi realizada ultrassonografia de vias urinárias que evidenciou nefrocalcinose. A paciente apresentou, ainda, FAN não reagente, fator reumatoide, $\mathrm{C} 3$ e $\mathrm{C} 4$ dentro dos valores da normalidade, bem como sorologia negativa para hepatite.

Foram necessários mais três internamentos por episódios de paralisia hipocalêmica (fraqueza intensa em membros inferiores, evoluindo com impossibilidade de movimentação dos mesmos e, posteriormente, do corpo inteiro), associado a extrassístoles ventriculares, devido a não aderência ao tratamento. Os exames laboratoriais desse período identificaram variação do potássio sério de 2,2 a 3,4 mmol/L, bicarnonato de 15,4 a 21,6 mmol/L, pH sérico de 7,24 a 7,33 e pH urinário de 7,0 a 7,5. Em tomografia computadorizada realizada em 2008, foi evidenciada nefrolitíase bilateral. Após cinco anos, paciente passou a referir dor em membro inferior direito devido à fratura de fêmur. Realizou densitometria óssea que evidenciou osteoporose.

\section{DISCUSSÃO}

A ATRd foi inicialmente descrita em 1946 por Albright et al. como sendo uma síndrome clínica caracterizada por hipocalemia, acidose metabólica hiperclorêmica, incapacidade de reduzir o $\mathrm{pH}$ urinário abaixo de 5,5 apesar da acidose sistêmica, nefrocalcinose e nefrolitíase. ${ }^{6} \mathrm{O}$ termo distal devese ao fato do gradiente entre $\mathrm{o} \mathrm{pH}$ urinário e sanguíneo ser estabelecido no néfron distal. ${ }^{6}$

A ATRd pode ser primária, devido a defeitos genéticos nos mecanismos de transporte dos túbulos renais, ou secundária, também denominada de adquirida. ${ }^{1}$ A forma genética pode ser tanto autossômica dominante quanto autossômica recessiva. ${ }^{6}$ Já a forma adquirida pode ocorrer devido a drogas (tratamento com anfotericina B, toxicidade pelo tolueno), infecções (hepatite, pielonefrite crônica) ou doenças auto-imunes (síndrome de Sjögren, lúpus eritematoso sistêmico). ${ }^{6}$

Essa patologia ocorre com mais frequência em pacientes com mais de 18 anos e no sexo feminino. ${ }^{6} \mathrm{O}$ caso apresentado corrobora com esse dado, tendo em vista se tratar de uma paciente do sexo feminino que apresentou os primeiros sintomas aos 18 anos. 
A ATRd possui baixa incidência na população em geral. ${ }^{7}$ Nessa patologia, a capacidade de acidificar a urina é perdida devido a um defeito na excreção de $\mathrm{H}+$ e $\mathrm{NH} 4+$ pelas células do ducto coletor. ${ }^{7} \mathrm{Na}$ ATRd, o pH urinário está acima de 6,0 na presença de acidose metabólica sistêmica ( $\mathrm{pH}$ sérico abaixo de 7,35). ${ }^{7}$ A paciente do caso, ao longo de seu acompanhamento, apresentou variação do pH sérico de 7,24 a 7,33 (com o ânion gap normal, em torno de seis) e do $\mathrm{pH}$ urinário de 7,0 a 7,5. A gasometria arterial evidencia, ainda, um valor limítrofe de pCO2, assinalando uma tentativa de compensação da acidose através do componente respiratório.

Como a excreção renal é a principal forma de eliminar ácido do corpo, há consequentemente uma tendência à acidose sistêmica. ${ }^{6}$ Esse acúmulo de ácidos no néfron distal leva ao consumo de bicarbonato e $\mathrm{CO} 2$, acarretando redução nos seus níveis sanguíneos. ${ }^{7}$

A hipocalemia ocorre com frequência na ATRd. ${ }^{4}$ Entretanto, raramente, pode se apresentar como fraqueza e paralisia muscular decorrente da perda de potássio, ${ }^{7}$ como ocorreu com nossa paciente. Esses sintomas, que caracterizam a paralisia hipocalêmica, estão relacionados à hipocalemia severa. ${ }^{8}$ No caso apresentado, a paciente possui paralisia periódica hipocalêmica tendo em vista que apresentou ataques recorrentes de fraqueza e paralisia muscular na vigência de hipocalemia. $^{5}$

Considera-se hipocalemia quando o potássio sérico está abaixo de 3,5 mEq/L7. No caso, a variação do potássio sérico da paciente foi de 2,2 a $3,4 \mathrm{mmol} / \mathrm{L}$. A hipocalemia deve-se, provavelmente, ao aumento da excreção de potássio na urina devido à incapacidade de secretar íons hidrogênio no néfron distal a fim de manter a eletroneutralidade na membrana apical, à diminuição da reabsorção tubular proximal em face da acidose e da hipocapnia, e à estimulação da aldosterona. ${ }^{2,6}$

Além das manifestações acima descritas, a hipocalemia pode acarretar distúrbios elétricos no miocárdio, sendo a arritmia cardíaca induzida pela hipocalemia a principal causa de óbito em pacientes com ATRd. ${ }^{6}$ Nossa paciente apresentou alguns episódios de extrassístoles assintomáticas, necessitando de internamento hospitalar para reposição endovenosa de potássio. Em alguns casos, a hipocalemia pode levar ainda à parada respiratória. ${ }^{2}$

Nossa paciente fez uso de: citrato de potássio, cloreto de potássio, carbonato de cálcio/vitamina $\mathrm{D}$, espironolactona e bicarbonato de sódio. O principal objetivo no tratamento da ATRd é corrigir a acidose. ${ }^{2}$ Estudos indicam que o citrato de potássio associado à vitamina $\mathrm{D}$ ativa corrigem a acidose metabólica, a hipocalemia e a hipofosfatemia, prevenindo a nefrocalcinose, a nefrolitíase e a osteoporose. ${ }^{2,9}$ A reposição de bicarbonato de sódio é bem aceita em pacientes que apresentam perda desse componente contribuindo para a acidose metabólica. ${ }^{10}$ Todavia, apesar dessa conduta aumentar o $\mathrm{pH}$ sérico, deve-se focar o tratamento na causa da acidose. ${ }^{10}$

A paciente do caso apresentou fratura em fêmur direito, sendo evidenciada osteoporose em densitometria óssea. A osteoporose está relacionada à acidose metabólica crônica da ATRd. ${ }^{6}$ Isso ocorre porque a acidose metabólica crônica acarreta diminuição na reabsorção tubular de cálcio, levando à hipercalciúria e ao hiperparatireoidismo leve. ${ }^{8,11}$ Nossa paciente chegou a apresentar calciúria de $516 \mathrm{mg} / 24 \mathrm{~h}$. Vale salientar que o tamponamento ósseo relacionado à carga ácida metabólica diária também favorece a hipercalciúria, a redução da massa óssea e a osteoporose. ${ }^{11}$

A ATRd ainda pode cursar com deposição de cálcio no tecido renal, levando à nefrocalcinose, e com formação de cálculos no trato urinário, levando à nefrolitíase. ${ }^{7}$ Há evidências de que a hipercalciúria em associação com a urina alcalina e os baixos níveis de citrato urinário contribuem para esse processo, favorecendo a nefrocalcinose e a nefrolitíase. ${ }^{11} \mathrm{~A}$ paciente evoluiu com nefrocalcinose e nefrolitíase bilateral evidenciadas em exames de imagens.

Se não for tratada, a ATRd acarreta atraso do crescimento e raquitismo em crianças e osteomalácia em adultos, sendo responsável, ainda, por deterioração da função renal ao longo dos anos. ${ }^{7}$ De fato, estudos indicam que pacientes com doença de longa duração apresentam com mais frequência disfunção renal. ${ }^{12}$ Todavia, se o diagnóstico for precoce e o tratamento for contínuo com bicarbonato de sódio e citrato de potássio, pode-se prevenir a progressão da doença, e os pacientes em geral apresentam um bom prognóstico., ${ }^{3,7}$ Infelizmente, nossa paciente apresentou grande dificuldade em aderir ao tratamento proposto, sobretudo por questões econômicas.

\section{CONCLUSÃO}

A ATRd é uma patologia de baixa incidência populacional, sendo a hipocalemia bastante associada ao quadro. Todavia, a paralisia hipocalêmica tem sido descrita em poucos casos. Dentre os principais sintomas que compreendem a síndrome, destacam-se ainda acidose metabólica, $\mathrm{pH}$ urinário inadequadamente alto, dano ósseo, nefrocalcinose e nefrolitíase. Todos esses sintomas foram apresentados no caso clínico acima relatado.

É necessário que, ao se deparar com um paciente que apresente paralisia periódica hipocalêmica, investigue-se ATRd, tendo em vista que o tratamento efetivo pode controlar a sintomatologia, levando a melhora significativa na qualidade de vida. O grande desafio consiste em garantir que o paciente seja capaz de seguir as recomendações médicas, fazendo adequado uso das medicações prescritas. 


\section{REFERÊNCIAS}

1. Almeida LP, Scrignoli JA, Penedo CT, Neves AC, Pinheiro MM, Ribeiro SL. Fratura femoral bilateral secundária à osteomalácia grave em paciente com acidose tubular renal tipo II. Acta Reumatol Port. 2010;35(4):508-12.

2. Both T, Zietse R, Hoorn EJ, van Hagen PM, Dalm VA, van Laar JA, van Daele PL. Everything you need to know about distal renal tubular acidosis in autoimmune disease. Rheumatol Int. 2014;34(8):1037-45.

3. Bhat MA, Laway BA, Mustafa F, Kuchay MS, Mubarik I, Palla NA. Hypokalemic quadriparesis and rhabdomyolysis as a rare presentation of distal renal tubular acidosis. Med J Islam Repub Iran. 2014;28(35):1-5.

4. Sunder S, Sathi S, Venkataramanan K, Verma H, Bhardwaj M, Rajesh J, Mahapatra H. A rare case of type I renal tubular acidosis with membranous nephropathy presenting as hypokalemic paralysis. Case Rep Nephrol Urol. 2013;3(2):91-8.

5. Garg RK, Malhotra HS, Verma R, Sharma P, Singh MK. Etiological spectrum of hypokalemic paralysis: a retrospective analysis of 29 patients. Ann Indian Acad Neurol. 2013;16(3):365-70.

6. Basak RC, Sharkawi KM, Rahman MM, Swar MM. Distal renal tubular acidosis, hypokalemic paralysis, nephrocalcinosis, primary hypothyroidism, growth retardation, osteomalacia and osteoporosis leading to pathological fracture: a case report. Oman Med J. 2011;26(4):271-74.
7. Escobar L, Mejía N, Gil H, Santos F. Distal renal tubular acidosis: a hereditary disease with an inadequate urinary $\mathrm{H}+$ excretion. Nefrología. 2013;33(3):289-96.

8. Okechuku G, Upadhyay K. An unusual case of recurrent hypokalemic paralysis in an adolescent: questions and answers. Pediatr Nephrol. 2015;30(9):1437-41.

9. Liao CY, Wang CC, Chen IH, Shiang JC, Liu MY, Tsai MK. Hypokalemic paralysis as a presenting manisfestation of primary Sjögren's syndrome accompanied by vitamin D deficiency. Intern Med. 2013;52(20):2351-3.

10. Andany MM, Fernández CF, Bayolo DM, Quintela EC, Montero AD. Sodium bicarbonate therapy in patients with metabolic acidosis. The Scientific World Journal. 2014:1-13.

11. Müller CS, Rachid A Filho, Carvalho JG, Freitas SS, Parchen CFR. Paralisia periódica hipocalêmica na síndrome de Sjögren. Rev Bras Reumatol. 2006;46(4):304-8.

12. Jha R, Muthukrishnan J, Shiradhonkar S, Patro K, Harikumar KV, Modi KD. Clinical profile of distal renal tubular acidosis. Saudi J Kidney Dis Transpl. 2011;22(2):261-7.

\section{Como citar:}

Daniel EC, Daher EF. Acidose tubular renal distal associada à Paralisia periódica hipocalêmica. Rev Med UFC. 2015 jul-dez;55(2):50-53. 